\title{
EFEITO DAS VARIAÇÕES ESPAÇO-TEMPORAIS DOS PARÂMETROS DE KOSTIAKOV-LEWIS NA INFILTRAÇÃO ACUMULADA ${ }^{1}$
}

\author{
Joaquim José de Carvalho², Carlos Alberto Vieira de Azevedo³, Everaldo Mariano Gomes \\ Jacqueline Henrique $^{2}$ \& José Dantas Neto ${ }^{3}$
}

\begin{abstract}
RESUMO
Avaliaram-se os efeitos das variações espacial e temporal dos parâmetros da equação de Kostiakov-Lewis, na estimativa da infiltração acumulada. Foram realizadas avaliações de campo num sistema de irrigação por sulco, para cada evento de irrigação ao longo da estação de cultivo, durante a qual foram observadas variações espaciais máximas de 94,7, 44,3 e 55,2\% e, espacialmente, as variações temporais máximas foram $80,0,42,5$ e $43,8 \%$, respectivamente, nos parâmetros $f o, k$ e $a$. Constatou-se, que quando fo, $k$ e $a$ variaram, respectivamente, de 0,000033 a $0,000419 \mathrm{~m}^{3} \mathrm{~min}^{-1} \mathrm{~m}^{-1}$, de 0,00438 a $0,02497 \mathrm{~m}^{3} \mathrm{~min}^{-1} \mathrm{~m}^{-1} \mathrm{e}$ de 0,000 a 0,419 , os reflexos na infiltração acumulada, para um tempo de oportunidade de infiltração de $177 \mathrm{~min}$, foram de 0,047 a $0,115 \mathrm{~m}^{3} \mathrm{~m}^{-1}$, de $0,045 \mathrm{a} 0,127 \mathrm{~m}^{3} \mathrm{~m}^{-1} \mathrm{e}$ de 0,037 a $0,115 \mathrm{~m}^{3} \mathrm{~m}^{-1}$, respectivamente.
\end{abstract}

Palavras-chave: irrigação por sulco, infiltração, manejo de água para irrigação

\section{EFFECTS OF SPATIAL AND TEMPORAL VARIATIONS OF THE KOSTIAKOV-LEWIS PARAMETERS ON THE CUMULATIVE INFILTRATION}

\begin{abstract}
The effects of the spatial and temporal variations in the Kostiakov-Lewis equation parameters on the cumulative infiltration were evaluated. Furrow irrigation field evaluations were performed at each irrigation event along the crop season. During the crop season, maximum spatial variations equal to $94.7,44.3$ and $55.2 \%$ were observed, and, spatially, the maximum temporal variations were $80.0,42.5$ and $43.8 \%$, respectively, in the parameters $f o, k$ and $a$. When $f o, k$ and $a$ varied, respectively, from 0.000033 to $0.000419 \mathrm{~m}^{3} \mathrm{~min}^{-1} \mathrm{~m}^{-1}$, from 0.00438 to $0.02497 \mathrm{~m}^{3} \mathrm{~min}^{-1} \mathrm{~m}^{-1}$, and from 0.0 to 0.419 , the reflexes on the cumulative infiltration, for an infiltration opportunity time equal to $177 \mathrm{~min}$, were 0.047 to $0.115 \mathrm{~m}^{3} \mathrm{~m}^{-1}, 0.045$ to $0.127 \mathrm{~m}^{3} \mathrm{~m}^{-1}$, and 0.037 to $0.115 \mathrm{~m}^{3} \mathrm{~m}^{-1}$, respectively.
\end{abstract}

Key words: furrow irrigation, infiltration, water irrigation management

\section{INTRODUÇÃO}

A velocidade de infiltração determina o volume de água que penetra no solo nas unidades de área e tempo estabelecendo, assim, o potencial para escoamento superficial. Este é o parâmetro de campo que mais afeta a taxa de avanço d'água nos sulcos de irrigação (Walker \& Skogerboe, 1987) influenciando no comprimento de sulcos (Henrique et al., 1997b) e

\footnotetext{
${ }^{1}$ Extraído da dissertação de Mestrado do primeiro autor. Convênio UFPB-Embrapa/Algodão-Escola Agrotécnica Federal de Souza, PB

${ }^{2}$ M.Sc. em Irrigação e Drenagem, Rua Ana Azevedo 509, Palmeira, CEP 58102 - 320, Campina Grande, PB, Fone: (083) 3217654

${ }^{3}$ Ph.D. em Irrigação e Drenagem, DEAg-UFPB, Av. Aprígio Veloso 882, Bodocongó, CEP 58109 - 970, Campina Grande, PB, Telefax: (083) 310 1185, E-mail: cazevedo@deag.ufpb.br

${ }^{4}$ M.Sc. em Irrigação e Drenagem, Escola Agrotécnica Federal de Sousa, Rua Tancredo Neves s/n, Jardim Sorrilândia, CP 49, CEP 58805 - 970 , Sousa, PB, Fone: (083) 521 1218, Fax: (083) 5211618
} 
comprometendo sensivelmente o desempenho desses sistemas, no que diz respeito, principalmente, às perdas por percolação. Henrique (1996) e Henrique et al. (1997a) estudando o efeito das características de infiltração de um solo franco-arenoso no desempenho de um sistema de irrigação por sulco, verificaram que, quando a infiltração decresceu de 0,168 para $0,094 \mathrm{~m}^{3} \mathrm{~m}^{-1}, \mathrm{o}$ tempo gasto para a água avançar até o final da área decresceu 450 min, resultando num aumento da eficiência de aplicação, de 32,9 para 60,5\%; os autores recomendam, então, a realização de um monitoramento sazonal, em tempo real, dessa fase, por ser ela o fator que mais contribui para as perdas por percolação comprometendo, assim, a eficiência de aplicação.

Existem inúmeras equações que descrevem o processo de infiltração d'água no solo, mas Clemmens (1983) apud Scaloppi et al. (1995) recomenda o uso das equações de Kostiakov ou Kostiakov-Lewis para propósitos de dimensionamento, avaliação e manejo de irrigação, porque elas descrevem razoavelmente bem esse processo. Philip (1957) apud Camacho et al. (1997) faz menção de que a equação de infiltração mais simples (Kostiakov) prediz muito bem a infiltração d'água no solo apenas quando os tempos de infiltração são pequenos; por isso é que a equação de Kostiakov-Lewis foi concebida, introduzindo-se um termo de infiltração constante na expressão de Kostiakov para descrever, de forma adequada, a infiltração, quando os tempos forem muito longos. De acordo com Santana (1996) e Santana et al. (1997) os coeficientes da equação de Kostiakov-Lewis dependem, além da textura e da estrutura do solo, de sua condição física no momento da irrigação como, por exemplo, a existência de fendas e o conteúdo de umidade inicial; os coeficientes $k$ e fo expressam a taxa de variação na quantidade de água que se infiltra ao longo do tempo, enquanto o expoente descreve o grau de variação não-linear entre a lâmina infiltrada e o tempo de oportunidade de infiltração.

Segundo Smith \& Duke (1984) as características de infiltração d'água no solo são particularmente difíceis de serem medidas em campo, com precisão, porque seus valores, além de dependerem das propriedades físicas do solo, são muito influenciados pelas variabilidades espaciais e temporais nos parâmetros de campo inerentes à hidráulica da irrigação superficial. Rivera et al. (1995) afirmam que a dificuldade em se caracterizar a infiltração no solo é um grande problema no dimensionamento dos sistemas de irrigação por superfície, porque torna crítica a determinação da vazão e do tempo de aplicação d'água adequados. Enciso-Medina et al. (1998) reportam que a variação no teor de umidade inicial do solo contribui para a variação espacial e temporal da taxa de infiltração; eles afirmam, ainda, que a taxa de infiltração é por demais afetada pelo desenvolvimento de crostas (selamentos) impermeáveis na superfície.

Conforme Azevedo (1993) o dimensionamento e manejo dos sistemas de irrigação por superfície tornam-se complicados devido as dificuldades em se medir as variações espacial e temporal nos parâmetros de campo, especialmente nas características de infiltração do solo. De acordo com Warrick (1983) a infiltração medida diretamente por anel infiltrômetro é limitada, por tratar-se de um valor pontual, não representando, em geral, as características de infiltração em grandes áreas; um outro aspecto é que a maior parte desses infiltrômetros mede a infiltração apenas numa dimensão; no entanto, as condições de sulco de irrigação possuem no mínimo duas dimensões nas características de infiltração.
Busman (1987) reporta sobre uma outra forma de se determinar a infiltração, que é através de hidrogramas de entrada e saída em sulcos de irrigação; este método compara o fluxo de entrada com o de saída em um sulco e a vantagem desta técnica é a maior abrangência na representatividade das características de infiltração assim determinadas, além da facilidade e flexibilidade das medições do fluxo, sempre que se irrigar; por outro lado, sua grande limitação é que as informações para esta finalidade se tornam completamente disponíveis somente quando o solo atinge sua capacidade de infiltração básica, não havendo, então, a possibilidade de se conhecer as características de infiltração, mesmo ainda durante a fase de avanço, objetivando-se prognosticar o desempenho do sistema de irrigação e realizar, caso necessário, alterações nos parâmetros operacionais, tendo em vista um alto nível de desempenho.

Azevedo (1992) desenvolveu um modelo matemático computacional, denominado SIRTOM (Surface Irrigation RealTime Optimization Model) que determina as características de infiltração do solo, descritas pela equação de Kostiakov-Lewis, a partir do ajuste do avanço simulado ao avanço medido; esta determinação ocorre durante a fase de avanço, possibilitando promover-se melhorias no desempenho do sistema de irrigação, em tempo real hábil.

O objetivo desta pesquisa foi avaliar, para um solo francoarenoso, os efeitos das variações espacial e temporal dos parâmetros da equação de Kostiakov-Lewis na estimativa da infiltração acumulada, visando-se identificar tanto sua sensibilidade a essas variações como a amplitude de variação na magnitude desses parâmetros ao longo da área irrigada, durante a estação de cultivo. Com essas informações, acredita-se que se poderá facilitar a tomada de decisão no manejo do sistema de irrigação por sulco no tipo de solo em estudo.

\section{MATERIAL E MÉTODOS}

O trabalho foi desenvolvido num sistema de irrigação por sulco da estação experimental da Embrapa Algodão, localizada no perímetro irrigado de São Gonçalo, Souza, PB. A área experimental (parcela agrícola 8B) cultivada com algodão, apresenta classe textural tipo franco-arenosa, mede 0,62 ha, foi sistematizada com declividade de $0,004 \mathrm{~m} \mathrm{~m}^{-1}$ no sentido longitudinal e $0,001 \mathrm{~m} \mathrm{~m}^{-1}$ no sentido transversal. $\mathrm{O}$ sistema de irrigação possui 53 sulcos com $73 \mathrm{~m}$ de comprimento, espaçados de 1,6 m, e foi operado sob condições de fluxo reduzido, onde se utilizaram dois sifões durante a fase de avanço e um na fase de armazenamento.

Para se observar as variações espacial e temporal nos parâmetros da equação de Kostiakov-Lewis, foram realizadas avaliações de campo para cada evento de irrigação, ao longo da estação de cultivo (total de 12) nas quais se coletaram dados em seis sulcos eqüidistantes, de forma a abranger ao máximo essas variações ao longo de toda a área irrigada. Os parâmetros $k$ e $a$ da equação de infiltração de Kostiakov-Lewis foram determinados a partir do ajuste do avanço simulado ao medido, processado pelo modelo matemático computacional SIRTOM (Azevedo, 1992; Azevedo et al., 1997ab). Como requerimento de dados de entrada do modelo SIRTOM, foram feitas as seguintes mensurações de campo: vazão de entrada, fase de avanço completa e perfilometria da seção transversal de fluxo. Como 
esta pesquisa fazia parte de uma outra, em que se desejava obter o tempo de aplicação em tempo real hábil, foi adotado, como dado de entrada para o modelo SIRTOM, um valor para a velocidade de infiltração básica igual a $0,000190 \mathrm{~m}^{3} \mathrm{~min}^{-1} \mathrm{~m}^{-1}$, determinado por Santana (1996); no entanto, este parâmetro foi também determinado em campo, tendo-se para isto medido a vazão de saída.

As vazões de entrada e de saída foram medidas através de calhas Parshall modelos H26-1 e H26-2 e calhas WSC, modelo FEX 1-34; inicialmente, essas medições eram feitas a cada minuto, até se verificar que a altura de fluxo tornou-se praticamente constante e, a partir daí, elas passaram a ser feitas a cada 10 min; entretanto, quando ocorria qualquer alteração na altura de fluxo voltava-se, então, a fazê-las a cada minuto. Para se medir a fase de avanço da água foram colocadas dez estacas ao longo de cada sulco escolhido, distanciadas a cada 7,3 m, cronometrando-se o tempo de avanço acumulado em cada estaca. A velocidade de infiltração básica, fo, foi determinada quando a vazão de saída, no final do sulco, atingiu valor constante; a fo foi, então, igual à diferença entre a vazão aplicada no início do sulco e a que saía em seu final (valores esses constantes) dividida pelo comprimento do sulco. Para se obter um valor médio da seção transversal de fluxo em cada sulco, foram realizadas medições em três estações, eqüidistantes 18,25 $\mathrm{m}$ ao longo do sulco, utilizando-se um perfilômetro confeccionado pelo Laboratório de Engenharia de Irrigação e Drenagem do
Departamento de Engenharia Agrícola da UFPB. A tomada desses dados foi feita apenas quando a fase de recessão se completou; no entanto, durante a fase de armazenamento, quando o perfil superficial de fluxo tornou-se praticamente estável, foram colocadas pequenas estacas nessas estações demarcando-se, assim, a seção transversal de fluxo a ser medida posteriormente. Foi atribuído, à rugosidade da superfície do solo, um valor igual a 0,013, utilizado por Azevedo (1992) e Azevedo et al. (1997ab) por terem achado ser este valor o que mais contribuiu para minimizar as discrepâncias entre o tempo de avanço simulado e o medido.

Os dados obtidos experimentalmente foram analisados utilizando-se métodos de estatística descritiva, calculando-se a média aritmética, mediana, valores mínimo e máximo, amplitude total, intervalo de $95 \%$ de confiança (com desvio-padrão populacional desconhecido) e coeficiente de variação. E, para avaliar a hipótese de normalidade dos dados, utilizou-se o método de Kolmogorov-Smirnov a nível de 5\% de probabilidade (Costa Neto, 1997).

\section{RESULTADOS E DISCUSSÃO}

As Tabelas 1, 2 e 3 apresentam as variações espacial e temporal nas características de infiltração d'água num solo franco-arenoso. Verificou-se, através da análise da distribuição de freqüência dos dados, que tanto a velocidade de infiltração

Tabela 1. Variação espacial e temporal da velocidade de infiltração básica, fo $\mathrm{x} 10^{-3}\left(\mathrm{~m}^{3} \mathrm{~min}^{-1} \mathrm{~m}^{-1}\right)$ num solo franco-arenoso

\begin{tabular}{|c|c|c|c|c|c|c|c|c|c|c|c|c|c|}
\hline \multirow{2}{*}{\multicolumn{2}{|c|}{$\begin{array}{l}\text { Eventos de } \\
\text { Irrigação }\end{array}$}} & \multicolumn{5}{|c|}{ Sulcos de irrigação } & \multirow{2}{*}{$\begin{array}{l}\text { Média } \\
\text { espac. }\end{array}$} & \multicolumn{2}{|c|}{ Valor } & \multirow{2}{*}{$\begin{array}{l}\text { Amp. } \\
\text { total }^{\mathrm{b}}\end{array}$} & \multicolumn{2}{|c|}{ I. C. $(95 \%)^{\mathrm{c}}$} & \multirow{2}{*}{$\begin{array}{l}\mathrm{CV}^{\mathrm{f}} \\
(\%)\end{array}$} \\
\hline & & 01 & 03 & 04 & 05 & 06 & & Mín. & Máx. & & L. I. $^{d}$ & L. S. $^{\mathrm{e}}$ & \\
\hline \multicolumn{2}{|c|}{01} & 0,214 & 0,345 & 0,140 & 0,247 & 0,123 & 0,214 & 0,123 & 0,345 & 0,222 & 0,103 & 0,325 & 41,8 \\
\hline \multicolumn{2}{|c|}{02} & 0,173 & 0,140 & $*$ & 0,132 & 0,156 & 0,150 & 0,132 & 0,173 & 0,041 & 0,121 & 0,179 & 12,1 \\
\hline \multicolumn{2}{|c|}{03} & 0,132 & 0,082 & 0,057 & 0,140 & 0,173 & 0,117 & 0,057 & 0,173 & 0,116 & 0,059 & 0,175 & 40,0 \\
\hline \multicolumn{2}{|c|}{04} & 0,156 & 0,099 & 0,041 & 0,107 & 0,197 & 0,120 & 0,041 & 0,197 & 0,156 & 0,046 & 0,194 & 49,4 \\
\hline \multicolumn{2}{|c|}{05} & 0,099 & 0,041 & 0,107 & 0,123 & 0,197 & 0,113 & 0,041 & 0,197 & 0,156 & 0,044 & 0,183 & 49,4 \\
\hline \multicolumn{2}{|c|}{06} & 0,156 & $*$ & 0,082 & 0,156 & 0,214 & 0,152 & 0,082 & 0,214 & 0,132 & 0,066 & 0,238 & 35,6 \\
\hline \multicolumn{2}{|c|}{07} & 0,156 & 0,066 & 0,107 & 0,132 & 0,197 & 0,132 & 0,066 & 0,197 & 0,131 & 0,070 & 0,193 & 37,6 \\
\hline \multicolumn{2}{|c|}{08} & 0,123 & $*$ & 0,082 & 0,140 & 0,189 & 0,134 & 0,082 & 0,189 & 0,107 & 0,063 & 0,204 & 33,2 \\
\hline \multicolumn{2}{|c|}{09} & $*$ & * & $*$ & 0,140 & 0,419 & 0,280 & 0,140 & 0,419 & 0,279 & $-1,493$ & 2,052 & 70,6 \\
\hline \multicolumn{2}{|c|}{10} & 0,057 & * & 0,041 & 0,173 & 0,370 & 0,160 & 0,041 & 0,370 & 0,329 & $-0,081$ & 0,402 & 94,7 \\
\hline \multicolumn{2}{|c|}{11} & 0,033 & $*$ & 0,041 & 0,205 & 0,271 & 0,138 & 0,033 & 0,271 & 0,238 & $-0,052$ & 0,327 & 86,7 \\
\hline \multicolumn{2}{|c|}{12} & 0,090 & 0,115 & 0,099 & 0,230 & 0,403 & 0,187 & 0,090 & 0,403 & 0,313 & 0,022 & 0,353 & 71,0 \\
\hline \multicolumn{2}{|c|}{$\begin{array}{c}\text { Média } \\
\text { temporal }\end{array}$} & 0,126 & 0,127 & 0,080 & 0,160 & 0,242 & 0,152 & & & & & & \\
\hline \multirow{2}{*}{$\frac{\overrightarrow{0}}{\stackrel{\pi}{J}}$} & Mín. & 0,033 & 0,041 & 0,041 & 0,107 & 0,123 & & 0,033 & & & & & \\
\hline & Máx. & 0,214 & 0,345 & 0,140 & 0,247 & 0,419 & & & 0,419 & & & & \\
\hline \multicolumn{2}{|c|}{ Amp. total } & 0,181 & 0,304 & 0,099 & 0,140 & 0,296 & & & & 0,386 & & & \\
\hline \multirow{2}{*}{ 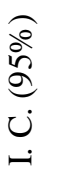 } & L. I. & 0,091 & 0,033 & 0,055 & 0,132 & 0,179 & & & & & 0,128 & & \\
\hline & L. S. & 0,162 & 0,221 & 0,104 & 0,189 & 0,0306 & & & & & & 0,177 & \\
\hline
\end{tabular}

\section{$\mathrm{CV}$}

$(\%)$

$42,0 \quad 80,0$

$42,9 \quad 27,6 \quad 41,3$

57,9

*Valor não obtido devido infiltrações laterais, ${ }^{\mathrm{a} M e ́ d i a ~ e s p a c i a l, ~}{ }^{\mathrm{b}} \mathrm{Amplitude}$ total, ${ }^{\mathrm{c} E s t i m a t i v a ~ d o ~ i n t e r v a l o ~ a ~} 95 \%$ de confiança, ${ }^{\mathrm{D}}$ Limite inferior, ${ }^{\mathrm{e}}$ Limite superior, ${ }^{\mathrm{f}}$ Coeficiente de variação. A quantificação dos parâmetros estatísticos na diagonal corresponde à variação de $f o$ ao longo do tempo e do espaço. Os valores de $f o$ nesta tabela deverão ser multiplicados por $10^{-3}$. 
Tabela 2. Variação espacial e temporal do coeficiente $\mathrm{k} \mathrm{x} 10^{-2}\left(\mathrm{~m}^{3} \mathrm{~min}^{-1} \mathrm{~m}^{-1}\right)$ da equação de Kostiakov-Lewis num solo franco-arenoso

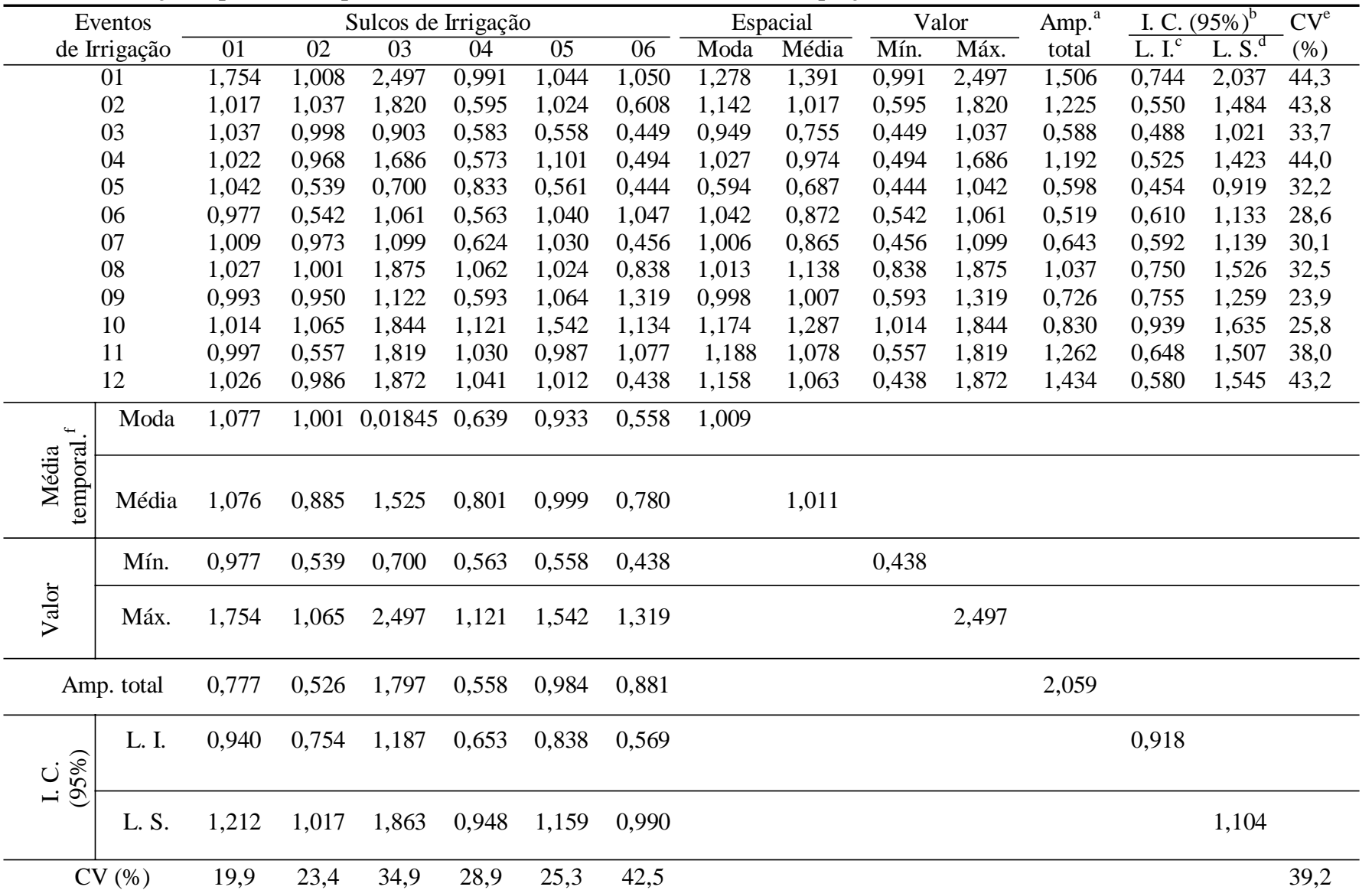

${ }^{\mathrm{a}}$ Amplitude total, ${ }^{\mathrm{b}}$ Estimativa do intervalo a 95\% de confiança, ${ }^{\mathrm{c}}$ Limite inferior, ${ }^{\mathrm{d}}$ Limite superior, ${ }^{\mathrm{e}}$ Coeficiente de variação, ${ }^{\mathrm{f}}$ Temporal, A quantificação dos parâmetros estatísticos na diagonal corresponde à variação do coeficiente $\mathrm{k}$ ao longo do tempo e do espaço. Os valores de $\mathrm{k}$ nesta tabela deverão ser multiplicados por $10^{-2}$

Tabela 3. Variação espacial e temporal do expoente a da equação de Kostiakov-Lewis num solo franco-arenoso

\begin{tabular}{|c|c|c|c|c|c|c|c|c|c|c|c|c|c|c|}
\hline \multirow{2}{*}{\multicolumn{2}{|c|}{$\begin{array}{c}\text { Eventos de } \\
\text { irrigação }\end{array}$}} & \multicolumn{6}{|c|}{ Sulcos de irrigação } & \multirow{2}{*}{$\begin{array}{c}\text { Média } \\
\text { espacial }\end{array}$} & \multicolumn{2}{|c|}{ Valor } & \multirow{2}{*}{$\begin{array}{c}\text { Amp. } \\
\text { total }\end{array}$} & \multicolumn{2}{|c|}{ I. C. $(95 \%)^{\mathrm{b}}$} & \multirow{2}{*}{$\begin{array}{l}\mathrm{CV}^{\mathrm{e}} \\
(\%)\end{array}$} \\
\hline & & 01 & 02 & 03 & 04 & 05 & 06 & & Mín. & Máx. & & L. I. $^{c}$ & L. S. $^{d}$ & \\
\hline \multicolumn{2}{|c|}{02} & 0,306 & 0,269 & 0,142 & 0,241 & 0,291 & 0,127 & 0,229 & 0,127 & 0,306 & 0,179 & 0,149 & 0,310 & 33,5 \\
\hline \multicolumn{2}{|c|}{04} & 0,299 & 0,352 & 0,180 & 0,297 & 0,235 & 0,171 & 0,256 & 0,171 & 0,352 & 0,181 & 0,180 & 0,332 & 28,3 \\
\hline \multicolumn{2}{|c|}{05} & 0,283 & 0,417 & 0,124 & 0,262 & 0,352 & 0,206 & 0,274 & 0,124 & 0,417 & 0,293 & 0,165 & 0,383 & 37,9 \\
\hline \multicolumn{2}{|c|}{06} & 0,340 & 0,419 & 0,269 & 0,324 & 0,282 & 0,262 & 0,316 & 0,262 & 0,419 & 0,157 & 0,254 & 0,378 & 18,7 \\
\hline \multicolumn{2}{|c|}{09} & 0,359 & 0,397 & 0,222 & 0,281 & 0,260 & 0,000 & 0,253 & 0,000 & 0,397 & 0,397 & 0,106 & 0,400 & 55,2 \\
\hline \multicolumn{2}{|c|}{10} & 0,321 & 0,269 & 0,227 & 0,203 & 0,275 & 0,213 & 0,251 & 0,203 & 0,321 & 0,118 & 0,204 & 0,299 & 17,9 \\
\hline \multicolumn{2}{|c|}{11} & 0,344 & 0,369 & 0,224 & 0,279 & 0,376 & 0,233 & 0,304 & 0,224 & 0,376 & 0,152 & 0,233 & 0,375 & 22,3 \\
\hline \multicolumn{2}{|c|}{12} & 0,302 & 0,356 & 0,207 & 0,258 & 0,322 & 0,206 & 0,275 & 0,206 & 0,356 & 0,150 & 0,210 & 0,340 & 22,5 \\
\hline \multicolumn{2}{|c|}{$\begin{array}{l}\text { Média } \\
\text { temporal }\end{array}$} & 0,312 & 0,355 & 0,197 & 0,265 & 0,303 & 0,181 & 0,269 & & & & & & \\
\hline$\frac{\bar{b}}{\frac{\pi}{\pi}}$ & Máx. & 0,359 & 0,419 & 0,269 & 0,324 & 0,376 & 0,273 & & & 0,419 & & & & \\
\hline \multicolumn{2}{|c|}{ Amp. total } & 0,079 & 0,150 & 0,183 & 0,121 & 0,141 & 0,273 & & & & 0,419 & & & \\
\hline \multirow{2}{*}{ 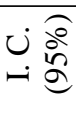 } & L. I. & 0,296 & 0,322 & 0,162 & 0,245 & 0,276 & 0,131 & & & & & 0,250 & & \\
\hline & L. S. & 0,329 & 0,387 & 0,231 & 0,285 & 0,329 & 0,231 & & & & & & 0,287 & \\
\hline \multicolumn{2}{|c|}{$\mathrm{CV}(\%)$} & 8,2 & 14,6 & 27,5 & 12,0 & 13,8 & 43,8 & & & & & & & 29,5 \\
\hline
\end{tabular}


básica, fo (Tabela 1) quanto o expoente $a$ da equação de Kostiakov-Lewis (Tabela 3) apresentaram, ao longo do tempo e do espaço, distribuição normal, enquanto o coeficiente $k$ (Tabela 2) não se ajustou a uma distribuição normal nem lognormal, indicando que a média aritmética e a mediana, por serem medidas de tendência central, não representam bem a distribuição deste coeficiente; logo, a moda, por ser uma medida de posição que indica a região de maior concentração de freqüências na distribuição, a representa melhor.

Através do coeficiente de variação (CV) observa-se que a variação espacial na velocidade de infiltração básica e nos parâmetros $k$ e $a$ da equação de Kostiakov-Lewis, ao longo da estação de cultivo, oscilou de 12,1 a $94,7 \%$, de 23,9 a $44,3 \%$ e de 17,9 a $55,2 \%$, respectivamente, enquanto no sentido transversal da área irrigada, a variação temporal oscilou de 27,6 a 80,0\%, de 19,9 a $42,5 \%$ e de 8,2 a 43,8\%, respectivamente. De acordo com os limites de CV propostos por Warrick \& Nielsen (1980) pode-se asseverar que, ao longo do tempo, a variação espacial apresentada pela velocidade de infiltração básica foi de média $(12 \% \leq \mathrm{CV} \leq 60 \%)$ a alta $(\mathrm{CV}>60 \%)$ e pelos parâmetros $k$ e $a$ foi média; já no sentido transversal da área irrigada a variação temporal na velocidade de infiltração básica foi, também, de média a alta, no coeficiente $k$ foi média e no expoente $a$ de baixa a média $(\mathrm{CV}<12 \%)$.

Ao longo da estação de cultivo a maior desuniformidade na velocidade de infiltração básica, no coeficiente $k$ e no expoente $a$, ocorreu nos décimo, primeiro e nono eventos de irrigação, respectivamente, enquanto no sentido transversal da área irrigada a maior desuniformidade na velocidade de infiltração básica ocorreu no sulco de irrigação 03 , e nos parâmetros $k$ e $a$ no sulco de irrigação 06, o que pode ser confirmado observando-se que é nesses eventos e sulcos de irrigação que se tem os maiores coeficientes de variação e proporções entre a amplitude total e a média aritmética, com exceção do coeficiente $k$, onde o sulco 03 que apresentou a maior proporção entre a amplitude total e a média aritmética, e não o sulco 06 que, no entanto, apresentou maior coeficiente de variação; isto é admissível devido ao fato da distribuição de freqüência dos valores deste coeficiente não ter apresentado distribuição normal.

A acentuada heterogeneidade nos parâmetros da equação de Kostiakov-Lewis, verificada ao longo do sentido transversal da área irrigada, já era esperada porque a parcela agrícola está inserida num corpo aluvial. Segundo Oliveira (1998) a heterogeneidade na textura desses solos é inerente à sua gênese, como resultado de depósitos sucessivos de diferentes materiais, oriundos de diversos locais; além da distribuição e do acúmulo desses materiais na área, função da topografia, do manejo físico e do processo de lixiviação a que esta área é submetida ao longo do tempo dentre, ainda, outros fatores; também, devido ao fato deste solo ser de textura tendendo a grossa, as dificuldades para o estabelecimento de uma estrutura bem definida e satisfatória às culturas, são bem maiores que em solos de textura fina.

Ao longo do tempo, a taxa de infiltração básica foi diminuindo até o quinto evento de irrigação devido, provavelmente, a um rearranjo estrutural das partículas do solo, como consequiência do carreamento das partículas mais finas de um local para outro, preenchendo os espaços porosos e diminuindo a taxa de infiltração do solo (Castros \& Souza, 1998) e a uma umidade inicial maior (imediatamente anterior ao evento de irrigação) no perfil do solo. Nos últimos eventos de irrigação ocorreu tendência de aumento na velocidade de infiltração básica em virtude do baixo teor de umidade inicial, ocasionado por aumento no turno de rega. Nos sulcos 01,03 e 04 não foi possível determinar a velocidade de infiltração básica em alguns eventos de irrigação e no sulco de irrigação 02 não foi possível determiná-la durante toda estação de cultivo, devido à ocorrência de fluxos preferenciais laterais, ocasionado por transbordamento, fazendo com que a vazão de saída não se estabilizasse. Supõe-se que as desuniformidades nos parâmetros $k$ e $a$ sempre tenham ocorrido em função de alterações tanto na estrutura do solo como em seu conteúdo de umidade inicial, isto é, antes de cada evento de irrigação conforme, também, Santana (1996) e Santana et al. (1997).

Verifica-se que, por toda a estação de cultivo, os parâmetros $k$ e $f o$ apresentaram valores médios mais elevados no primeiro evento de irrigação e, em seguida, tenderam a decrescer ao longo do tempo até o sétimo evento de irrigação, enquanto o expoente $a$ apresentou tendência crescente até o respectivo evento de irrigação; portanto, no primeiro evento de irrigação a capacidade de infiltração d'água do solo foi maior, o que se deve a uma porosidade máxima total (ocasionada por um recente preparo de solo) e a um baixo teor de umidade inicial no solo, enquanto do segundo ao sétimo evento de irrigação o decréscimo da capacidade de infiltração se deve, principalmente, a um rearranjo estrutural do solo e a teores mais elevados de umidade existentes, ocasionados por turnos de rega menores e a aumentos, ao longo do tempo, da seção transversal dos sulcos de irrigação. A partir do oitavo evento de irrigação, a capacidade de infiltração d'água no solo tendeu a aumentar devendo-se, principalmente, a baixos teores de umidade existentes no solo, ocasionados por turnos de rega maiores. Esse comportamento sazonal dos parâmetros de Kostiakov-Lewis está coerente, porque $k$ e fo representam, respectivamente, as taxas de infiltração sob condições de insaturação e saturação, e $a$ descreve como a infiltração acumulada varia com o tempo. Se essas taxas diminuíram ao longo do tempo, o expoente $a$ teria realmente que crescer para refletir uma relação mais linear entre a infiltração acumulada e o tempo.

Ao longo do tempo, a média espacial da velocidade de infiltração básica (Tabela 1) e do expoente $a$ (Tabela 3 ) oscilou, respectivamente, de 0,000113 a $0,000280 \mathrm{~m}^{3} \mathrm{~min}^{-1} \mathrm{~m}^{-1}$ e de 0,229 a 0,316 e a moda espacial do coeficiente $k$ oscilou de 0,00594 a $0,01278 \mathrm{~m}^{3} \mathrm{~min}^{-1} \mathrm{~m}^{-1}$ (Tabela 2); já no sentido transversal da área irrigada, a média temporal da velocidade de infiltração básica e do expoente $a$ oscilou, respectivamente, de 0,000080 a 0,000242 $\mathrm{m}^{3} \mathrm{~min}^{-1} \mathrm{~m}^{-1}$ e de 0,181 a 0,355 e a moda temporal do coeficiente $k$ oscilou de 0,00558 a $0,01845 \mathrm{~m}^{3} \mathrm{~min}^{-1} \mathrm{~m}^{-1}$.

Ao longo do tempo e do espaço, para os parâmetros fo e $a$, a média, o coeficiente de variação e a estimativa do intervalo a 95\% de confiança (para os 53 sulcos de irrigação) foram, respectivamente, $0,000152 \mathrm{~m}^{3} \mathrm{~min}^{-1} \mathrm{~m}^{-1} \mathrm{e} 0,269,57,9 \%$ e $29,5 \%$, e de 0,000033 a $0,000419 \mathrm{~m}^{3} \mathrm{~min}^{-1} \mathrm{~m}^{-1}$, e de 0,000 a 0,419 ; para o coeficiente $k$ a moda, o coeficiente de variação e a estimativa do intervalo a $95 \%$ de confiança foram de $0,01009 \mathrm{~m}^{3} \mathrm{~min}^{-1} \mathrm{~m}^{-1}$, $39,2 \%$ e de 0,00438 a $0,02497 \mathrm{~m}^{3} \mathrm{~min}^{-1} \mathrm{~m}^{-1}$, respectivamente.

A Tabela 3 registra um fato inusitado detectado nesta análise; para o nono evento de irrigação no sulco 06 , a técnica de solução do problema inverso da irrigação por sulco, utilizada nesta pesquisa, encontrou um valor nulo para o expoente $a$ sugerindo, assim, que o processo de infiltração sob condições de insaturação, descrito pela equação de Kostiakov-Lewis independe, neste caso, do tempo; isto se apresenta, a princípio, teoricamente impossível. Deve-se lembrar de que, na 
determinação das características de infiltração d'água no solo, o procedimento de ajuste do avanço simulado ao medido incorpora nos parâmetros de Kostiakov-Lewis, todas as peculiaridades da irrigação por sulco que influenciam na infiltração acumulada, inclusive o tempo de avanço.

Os dados das 72 avaliações de campo, realizadas ao longo da estação de cultivo, utilizados na obtenção dos parâmetros de Kostiakov-Lewis, mostram que o avanço d'água no final dos sulcos tornou-se, de forma generalizada, mais rápido do primeiro ao último sulco, isto é, na direção do fluxo no canal parcelar de condução d'água, refletindo principalmente uma diminuição na capacidade de infiltração d'água no solo, nesta direção. As Figuras 1A-C exibem o efeito da duração da fase de avanço nos parâmetros de Kostiakov-Lewis, nas quais se observa que, quando a fase avanço se torna mais rápida, os valores de $k$ e $a$ aumentam, enquanto os de fo diminuem, fato que demonstra, então, que nas condições de insaturação do solo realmente ocorreu, do primeiro ao último sulco, diminuição na capacidade de infiltração d'água; as condições de insaturação são as que mais afetam a fase de avanço. $\mathrm{O}$ valor nulo para $a$, encontrado para um tempo de avanço no final da área, de 16 min (o mais baixo de todos) significa que as condições de insaturação ocorreram num período muito curto, a ponto da infiltração acumulada nessas condições se tornar independente do tempo; entretanto, a outra fase do processo de infiltração (condições de saturação) foi dependente do tempo.

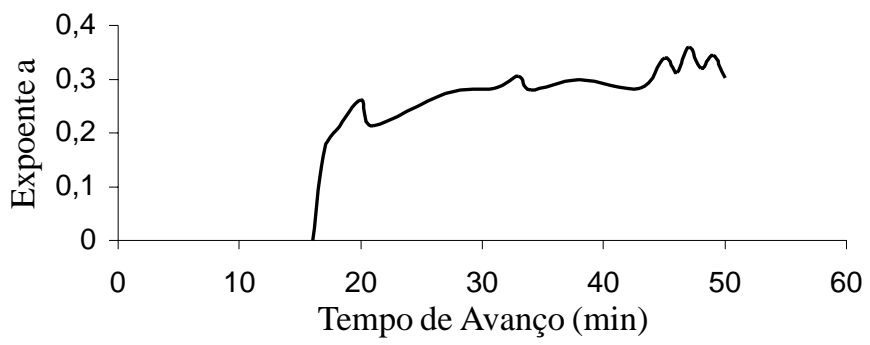

(A)

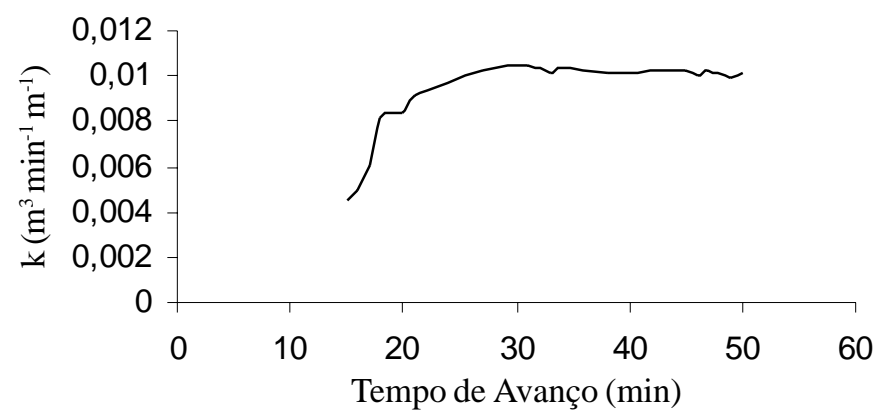

(B)

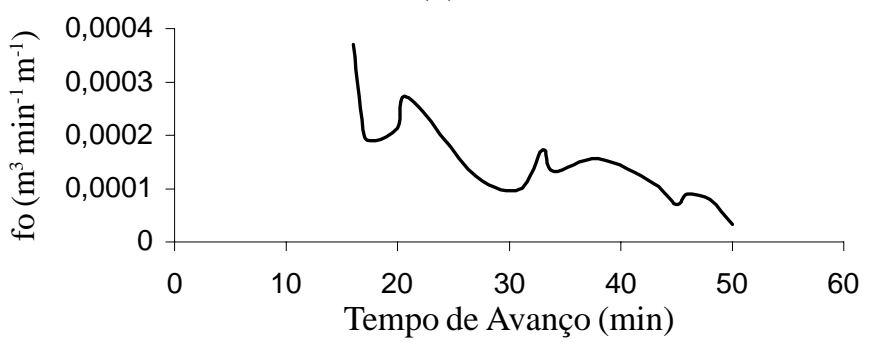

(C)

Figura 1. Efeito do tempo de avanço no final do sulco nos parâmetros $a(\mathrm{~A}), k(\mathrm{~B})$ e $f o(\mathrm{C})$
Dentre outros fatores, a infiltração acumulada depende do tempo de aplicação d'água que, na irrigação por sulco tem, como componentes, o tempo de avanço no final do sulco e o tempo necessário para se aplicar a lâmina desejada ao longo de toda área, razão porque os parâmetros de Kostiakov-Lewis, descrevendo o processo de infiltração na irrigação por sulco, têm que ser determinados por um procedimento que incorpore, neles, o efeito da duração da fase de avanço na infiltração acumulada. Os parâmetros de Kostiakov-Lewis, determinados a partir de dados de infiltração obtidos num teste (pontual) pelo método dos cilindros infiltrômetros exprimem tão somente os efeitos do tempo, da textura e da estrutura do solo numa infiltração que teve, inclusive, apenas uma componente (a vertical); esses parâmetros não descrevem adequadamente a infiltração na irrigação por sulcos. A grande maioria dos valores dos parâmetros de Kostiakov-Lewis apresentados na literatura, tem sido determinada a partir desses testes; por isto, não se torna fácil encontrar, na literatura, um valor nulo para $a$. Acredita-se, porém, que o fato inusitado acima evidenciado, esteja pelo menos parcialmente explicado dentro das possibilidades de introspecção que os dados desta pesquisa assim permitem.

As Figuras 2A-C exibem o efeito individual das variações dos parâmetros $f o, k$ e $a$, na estimativa da infiltração acumulada, respectivamente. Os valores da infiltração acumulada, apresentados na Figura 2A, foram calculados para valores constantes de $k, a$ e $\tau$ (tempo de oportunidade de infiltração) alterando-se, então, apenas os valores de fo dentro da faixa de variação encontrada em campo $\left(0,000033 \mathrm{a} 0,000419 \mathrm{~m}^{3} \mathrm{~min}^{-1} \mathrm{~m}^{-1}\right)$; na Figura 2B, para valores constantes de fo, $a$ e $\tau$, onde se alteraram apenas os valores do coeficiente $k$ dentro da faixa de variação medida em campo $\left(0,00438\right.$ a $\left.0,02497 \mathrm{~m}^{3} \mathrm{~min}^{-1} \mathrm{~m}^{-1}\right)$ e na Figura 2C, para valores constantes de $f o, k$ e $\tau$ alterando-se, assim, somente os valores do expoente $a$ na faixa de variação verificada em campo $(0,000$ a 0,419$)$. Os valores constantes, que representam a média global, isto é, ao longo do tempo e do espaço, usados para os parâmetros fo (Tabela 1), $k$ (Tabela 2), $a$ (Tabela 3) e $\tau$ foram de $0,000152 \mathrm{~m}^{3} \mathrm{~min}^{-1} \mathrm{~m}^{-1}, 0,01011 \mathrm{~m}^{3} \mathrm{~min}^{-1} \mathrm{~m}^{-1}$, 0,269 e $177 \mathrm{~min}$, respectivamente.

Isoladamente, constatou-se efeito linear significativo de cada parâmetro de Kostiakov-Lewis na estimativa da infiltração acumulada. Quando $f o, k$ e $a$ variaram, respectivamente, de 0,000033 a $0,000419 \mathrm{~m}^{3} \mathrm{~min}^{-1} \mathrm{~m}^{-1}$, de 0,00438 a $0,02497 \mathrm{~m}^{3} \mathrm{~min}^{-1} \mathrm{~m}^{-1}$ e de 0,000 a 0,419 , os reflexos na infiltração acumulada, para um tempo de oportunidade de infiltração de $177 \mathrm{~min}$, foram de 0,047 a $0,115 \mathrm{~m}^{3} \mathrm{~m}^{-1}$, de 0,045 a $0,127 \mathrm{~m}^{3} \mathrm{~m}^{-1}$ e de 0,037 a $0,115 \mathrm{~m}^{3} \mathrm{~m}^{-1}$, respectivamente. Observando-se que a amplitude com a qual a infiltração acumulada variou nas três situações foi praticamente a mesma fica demonstrado, então, que a infiltração acumulada é bastante sensível a fo, seguido do $k$, porque as grandezas absolutas com que fo varia são bem menores que as referentes aos parâmetros $k$ e $a$; realmente, era de se esperar que a infiltração acumulada fosse bem mais sensível ao $f o$ e ao $k$, porque esses parâmetros expressam as taxas de infiltração sob condições de saturação e insaturação, respectivamente, enquanto o parâmetro $a$ simplesmente descreve como a infiltração acumulada varia ao longo do tempo de oportunidade de infiltração, isto é, ele define o grau de não-linearidade nessa variação. Na condição de saturação, o meio poroso do solo está em sua capacidade máxima de contribuição para a infiltração, além de que o fluxo nessa 


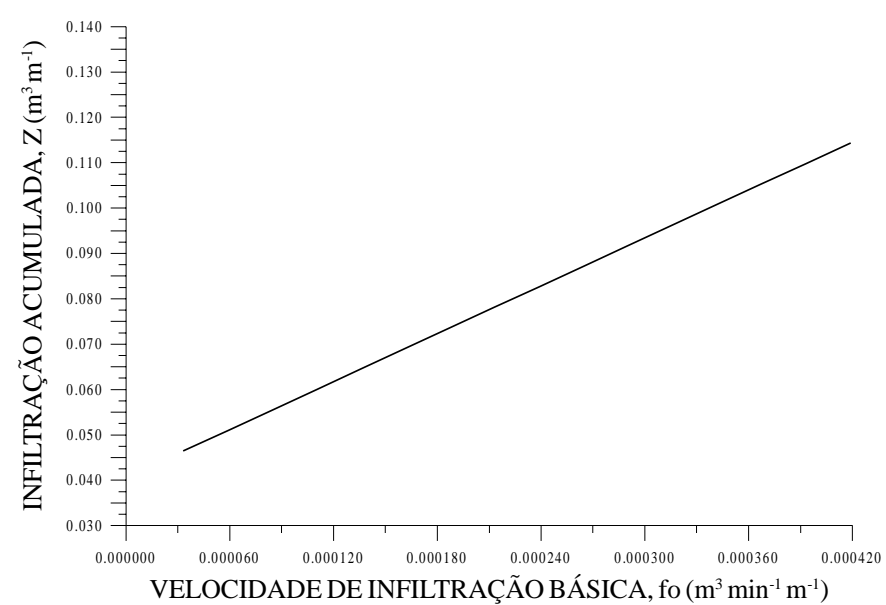

(A)

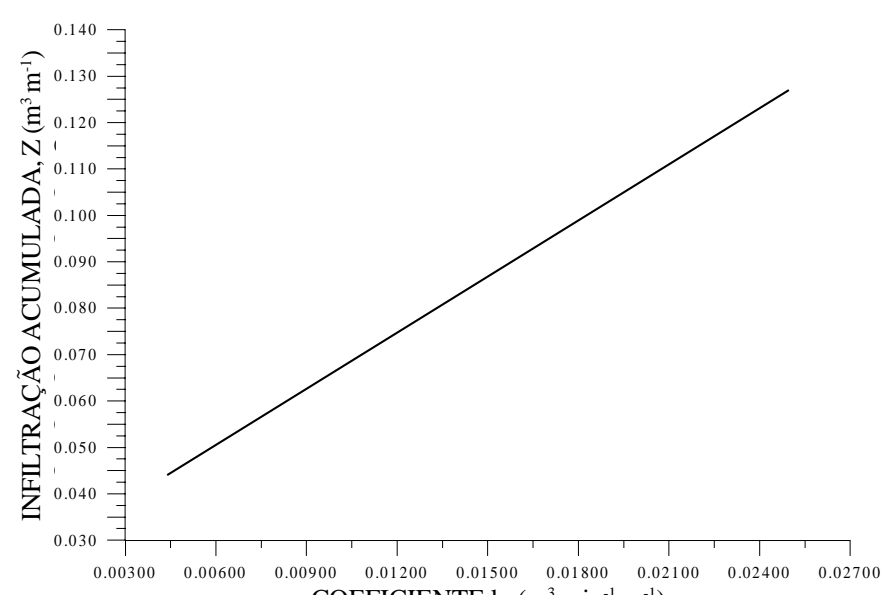

(B)

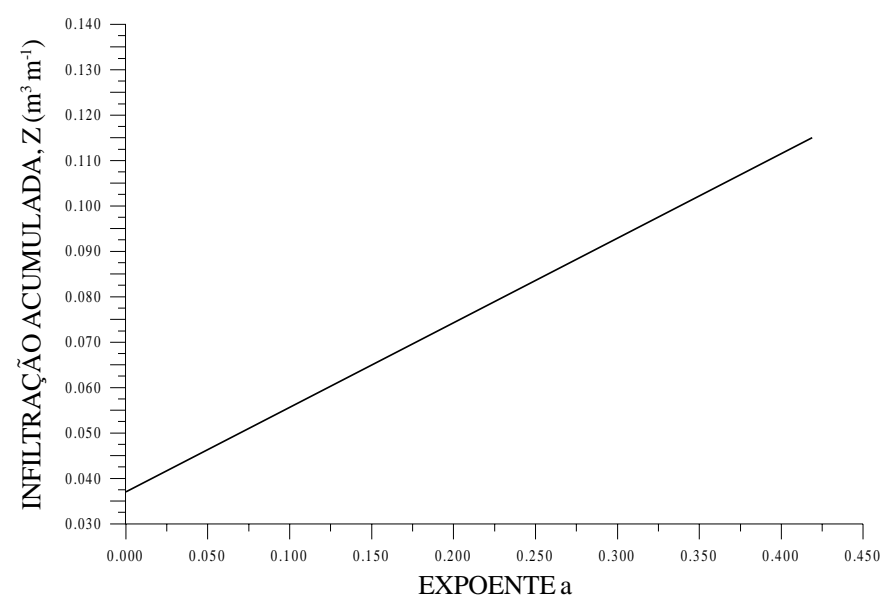

(C)

Figura 2. Efeito individual da variação dos parâmetros $f o$ (A), $k$ (B) e $a$ (C) na estimativa da infiltração acumulada

condição é bem mais duradouro que na condição de insaturação, em especial quando se deseja aplicar a lâmina d'água requerida para se estabelecer uma eficiência de armazenamento de 100\%; assim sendo, explica-se mais ainda porque a infiltração acumulada é extremamente sensível às grandezas de $f o$.

A Figura 3 exibe o efeito agregado das variações dos parâmetros $k$ e $a$ na estimativa da infiltração acumulada; cada barra, nesta figura, representa uma equação de Kostiakov-Lewis distinta; no
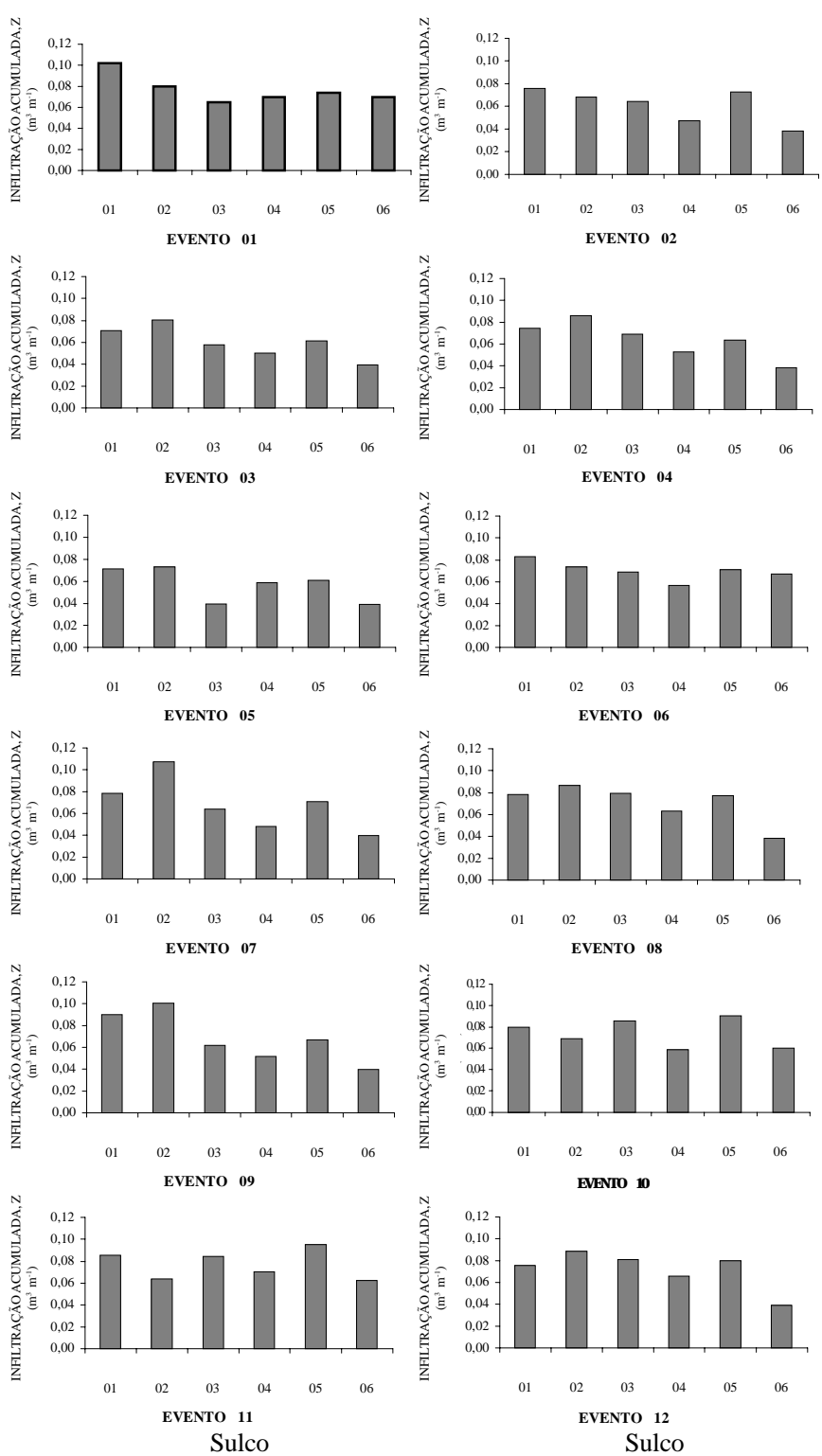

Figura 3. Efeito agregado da variação espacial e temporal de $k \mathrm{e}$ $a$ da equação de Kostiakov-Lewis na infiltração acumulada num solo franco-arenoso

entanto, deve-se enfatizar que os valores de $f o$ e $\tau$ (tempo de oportunidade de infiltração) foram mantidos constantes em todas as equações, tendo sido seus valores iguais, respectivamente, a $0,000152 \mathrm{~m}^{3} \mathrm{~min}^{-1} \mathrm{~m}^{-1}$ (Tabela 1) e 177 min que correspondem às médias ao longo do tempo e do espaço. Observa-se uma variação da infiltração acumulada tanto no tempo quanto no espaço, ocasionada pela variação espacial e temporal nos parâmetros $k$ e $a$. As variações na infiltração acumulada foram estudadas apenas sob o aspecto das variações nas taxas de infiltração d'água no solo, descritas pela equação de Kostiakov-Lewis; entretanto, sabese que a infiltração acumulada pode também variar em função de variações noutros parâmetros como, por exemplo, o tempo de aplicação d'água pelo sistema de irrigação e o tamanho da seção transversal de fluxo. Existe uma tendência muito forte da seção transversal de um sulco se alargar ao longo da estação de cultivo favorecendo, assim, a um volume maior d'água armazenado no sulco em que irá se infiltrar; fazem-se necessárias, portanto, pesquisas de campo que avaliem, de forma mais completa, as variações nos parâmetros que afetam a infiltração acumulada. 
Os resultados desta pesquisa demonstram a importância de se fazer um monitoramento espacial e temporal das características de infiltração d'água no solo, visando-se estabelecer um manejo do sistema que resulte num balanceamento melhor das perdas d'água por percolação e escoamento superficial favorecendo, assim, níveis satisfatórios de desempenho. O conhecimento, a priori, tanto da sensibilidade da infiltração acumulada às variações nos parâmetros de Kostiakov-Lewis como da amplitude de variação nesses parâmetros, ao longo da área irrigada e durante a estação de cultivo, poderá facilitar a tomada de decisão no manejo do sistema de irrigação por sulco no tipo de solo estudado.

\section{CONCLUSÕES}

1. Ao longo do tempo e do espaço, os valores mínimo e máximo da velocidade de infiltração básica $(f o)$, do coeficiente $k$ e do expoente $a$ da equação de Kostiakov-Lewis foram, respectivamente, de 0,000033 e $0,000419 \mathrm{~m}^{3} \mathrm{~min}^{-1} \mathrm{~m}^{-1}, 0,00438 \mathrm{e}$ $0,02497 \mathrm{~m}^{3} \mathrm{~min}^{-1} \mathrm{~m}^{-1}$, e de 0,000 e 0,419 . Por toda a estação de cultivo essas amplitudes geraram variações espaciais máximas iguais a 94,7, 44,3 e 55,2\% e, espacialmente, as variações temporais máximas foram de 80,0, 42,5 e 43,8\%, respectivamente, nos parâmetros fo, $k$ e $a$.

2. Constatou-se efeito linear significativo de cada parâmetro de Kostiakov-Lewis na infiltração acumulada; quando fo, $k$ e $a$ variaram, respectivamente, de 0,000033 a $0,000419 \mathrm{~m}^{3} \mathrm{~min}^{-1} \mathrm{~m}^{-1}$, de 0,00438 a $0,02497 \mathrm{~m}^{3} \mathrm{~min}^{-1} \mathrm{~m}^{-1}$ e de 0,000 a 0,419 , os reflexos na infiltração acumulada, para um tempo de oportunidade de infiltração de $177 \mathrm{~min}$, foram de 0,047 a $0,115 \mathrm{~m}^{3} \mathrm{~m}^{-1}$, de $0,045 \mathrm{a}$ $0,127 \mathrm{~m}^{3} \mathrm{~m}^{-1}$ e de 0,037 a $0,115 \mathrm{~m}^{3} \mathrm{~m}^{-1}$, respectivamente.

\section{REFERÊNCIAS BIBLIOGRÁFICAS}

AZEVEDO, C.A.V. de. Real-time solution of the inverse furrow advance problem. Logan: Utah State University, 1992. 263p. Ph.D. Thesis

AZEVEDO, C.A.V. de. Modelagem em sistemas de checagem e controle de irrigação por sulco. In: CONGRESSO BRASILEIRO DE ENGENHARIA AGRÍCOLA, 22, 1993, Anais... Ilhéus: SBEA, 1993.p. 2193-2208.

AZEVEDO, C.A.V.; WALKER, W.R.; MERKLEY, G.P. Modelo de ondas cinemáticas na solução do problema inverso da irrigação por sulco em tempo real. Revista Brasileira de Engenharia Agrícola e Ambiental, Campina Grande, v. 1, p. 35-41, 1997a.

AZEVEDO, C.A.V.; WALKER, W.R.; MERKLEY, G.P. Otimização não-linear na solução do problema inverso da irrigação por sulco em tempo real. Revista Brasileira de Engenharia Agrícola e Ambiental, Campina Grande, v. 1, p. 43-49, 1997 b.

BUSMAN, J.D. Optimizing control of surface irrigation using concurrent evaluation of infiltration. Logan, Utah: Utah State University, 1987. Dissertation

CAMACHO, E.; PÉREZ-LUCENA, C.; ROLDÁN-CAÑAS, J.; ALCAIDE, M. IPE: Model for management and control of furrow irrigation in real time. Journal of Irrigation and Drainage Engineering, ASCE, New York, v. 123, n. 4, p. 264-269. 1997.
CASTROS, L.C.A.; SOUZA, F. Análise dos parâmetros de infiltração na irrigação por sulcos utilizando a metodologia do balanço de volume. In: CONGRESSO BRASILEIRO DE ENGENHARIA AGRÍCOLA, 27, 1998, Poços de Caldas. Anais... Poços de Caldas: SBEA, 1998. p. 172-174.

COSTA NETO, P.L. de O. Estatística. 15 ed. São Paulo: Edgard Blücher, 1997. 264p.

ENCISO-MEDINA, J.; MARTIN, D.; EISENHAUER, D. Infiltration model for furrow irrigation. Journal of Irrigation and Drainage Engineering, ASCE, New York, v. 124, n. 2, p. 73-80. 1998.

HENRIQUE, J. Influência dos parâmetros de campo no desempenho dos sistemas de irrigação por sulco. Campina Grande: Universidade Federal da Paraíba, 1996. 66p. Dissertação Mestrado

HENRIQUE, J.; AZEVEDO, C.A.V.; BARRETO, A.N.; BEZERRA, J.R.C.; DANTAS NETO, J. Efeitos da infiltração no desempenho da irrigação por sulco simulada em solo franco-arenoso. In: CONGRESSO BRASILEIRO DE ENGENHARIA AGRÍCOLA, 26, 1997, Campina Grande. Anais... Campina Grande: SBEA, 1997a. CD ROM.

HENRIQUE, J.; AZEVEDO, C.A.V.; BARRETO, A.N.; BEZERRA, J.R.C.; DANTAS NETO, J. Efeitos do comprimento de sulcos no desempenho das irrigações simuladas em solo francoarenoso. In: CONGRESSO BRASILEIRO DE ENGENHARIA AGRÍCOLA, 26, 1997, Campina Grande. Anais... Campina Grande: SBEA, 1997b. CD ROM.

OLIVEIRA, J.J. de. Variabilidade espacial de características químicas em um solo salino-sódico. Campina Grande: Universidade Federal da Paraíba, 1998. 98p. Dissertação Mestrado

RIVERA, R.C.; CASTRO, L.C.A. de; SOUZA, F. de. Variação dos parâmetros de infiltração na irrigação por sulco ao longo do ciclo de desenvolvimento da cultura do feijão (Vigna unguiculata L. walp). In: CONGRESSO BRASILEIRO DE ENGENHARIA AGRÍCOLA, 24, 1995, Viçosa. Anais...Viçosa: SBEA, 1995.p. 206.

SANTANA, E.F. Monitoramento sazonal do desempenho de um sistema de irrigação por sulco no perímetro irrigado de São Gonçalo, PB. Campina Grande: Universidade Federal da Paraíba, 1996.96p. Dissertação Mestrado

SANTANA, E.F.; AZEVEDO, C.A.V.; BARRETO, A.N.; DANTAS NETO, J.; BEZERRA, J.R.C. Monitoramento temporal e sazonal da infiltração do sulco na cultura do algodoeiro. In: CONGRESSO BRASILEIRO DE ALGODÃO, 1, 1997, Fortaleza. Anais... Fortaleza: EMBRAPA Algodão, 1997. p. 372-375.

SCALOPPI, E.J.; MERKLEY, G.P.; WILLARDSON, L.S. Intake parameters from advance and wetting phases of surface irrigation. Journal of Irrigation and Drainage Engineering, ASCE, New York, v. 121, n. 1, p 57-70. 1995.

SMITH, D.L.; DUKE, H.R. Prediction of irrigation advance rates in real time. ASAE paper No. 84-2590. 1984.

WALKER, W.R.; SKOGERBOE, G.V. Surface Irrigation: Theory and practice. 1. New Jersey: Ed. Englewood Cliffs: Prentice-Hall INC, 1987.386p.

WARRICK, A.W. Parameters in infiltration equations. Advances in Infiltration. In: NATIONAL CONFERENCEON ADVANCES IN INFILTRATION, Proceedings... ASAE, 69-81. 1983.

WARRICK, A.W.; NIELSEN, D.R. Spatial variability of soil physical properties in the field. In: HILLEL, D. 1.ed. Application of soil physics. New York: Academic Press, 1980. cap. 13 , p. $319-344$. 